\title{
Tracing kinetic transformations of organomineral associations in a changing climate
}

Donald L. Sparks ${ }^{1 *}$, Elizabeth K. COWARD ${ }^{1}$, Tyler D.

SOWERS ${ }^{1,2}$, ElIZABETH J. TOMASZEWSKI ${ }^{1}$

${ }^{1}$ Delaware Environmental Institute, Univ. of Delaware,

Newark, DE, United States (*correspondence to

dlsparks@udel.edu)

${ }^{2}$ US Environmental Protection Agency, Research Triangle

Park, Durham, NC, United States

Despite the well-established importance of reactive mineral phases in organic carbon stabilization, the permanence of organomineral complexes is highly heterogeneous across broad spatial and temporal scales. Observed variability in turnover times is rooted in the diversity of kinetic mechanisms involved in organomineral association stability, such as molecular assembly and partitioning, co-precipitation and dissolution, exchange and desorption reactions. Mineral interfaces, not simply retentive active sites, should be considered as dynamic hotbeds of stabilization, transformation and release of organic compounds across biogeochemical environs. Much of our evolving understanding of organomineral interactions has been enabled by analytical advancements, which with extended spatial, and notably, temporal resolution, have allowed detection of such processes. We have leveraged ultrahigh-resolution mass spectrometry and paired synchrotron-based spectroscopic approaches to tracing kinetic organic transformations in situ. Employing such techniques, we have revealed the nanoscale formation of adsorptive molecular fractionations at iron (oxyhydr)oxide interfaces to macroscale redox-mediated release of organic compounds from minerals in vulnerable thawing permafrost and coastal wetland sediments impacted by rising sea level. Given the urgent need to move conceptual models of $\mathrm{C}$ stabilization beyond the theoretical, future applications of high-resolution techniques may acutely advance our understanding of organomineral plasticity - both complementary and competing reactions - in increasingly volatile systems. 